\title{
Special issue on deep learning and neural computing for intelligent sensing and control
}

\author{
Xiaomeng $\mathrm{Ma}^{1} \cdot$ Qingyuan Zhou ${ }^{2}$
}

Published online: 27 February 2020

(C) Springer-Verlag London Ltd., part of Springer Nature 2020

Intelligent sensing, especially together with autonomous decision making and control, recently has gained wide attention, with successful showcases in different areas such as the autonomous flying droids, self-driving cars, and Amazon-Kiva systems. One primary ultimate goal is that via active sensing, the computer/machine can learn through either supervised or unsupervised information to perform different tasks. This fact renders learning a fundamental component for both sensing and control. Among many learning approaches, deep learning has obtained a series of success across various domains including image, speech, text as well as various user interaction data. The resulting increased sensing capability opens up new possibility for more intelligent decision making and control. On the other hand, emerging technology, e.g., deep reinforcement learning and big data, also spurs the research for new control paradigm. The continuously increasing interest in the intersection between intelligent sensing, big data, and deep learning motivates us to organize this special section to study the learning of feature representations for decision-making and control problems.

The submitted manuscripts were reviewed by experts from both academia and industry. After two rounds of reviewing, the highest quality manuscripts were accepted for this special issue. This special issue will be published by Neural Computing and Applications as special issues. Totally, 19 papers are suggested to EiC for acceptance. The selected papers are summarized as follows.

In order to solve the problem of extracting a large amount of data in large-scale image analysis, a content

Xiaomeng Ma

xiaomengma@szu.edu.cn

1 College of Economics, Shenzhen University, 3688 Nanhai Ave, Nanshan Qu, Shenzhen 518060, China

2 School of Economics and Management, Changzhou Vocational Institute of Mechatronic Technology, 26 Mingxinzhong Road, Changzhou 213164, China semantic image content analysis and storage scheme based on intelligent computer learning image annotation is proposed by Wei et al. [1]. By analyzing the difference between capsule network and traditional convolutional neural network, it is found that the model compression method applied to the traditional neural network cannot be directly used in the capsule network. To address the problem, an IPC-CapsNet compression algorithm is proposed by Zhong et al. [2] based on the structural characteristics of the capsule networks. Jain et al. [3] propose a pipeline called deep refinement which utilizes some of the state-of-the-art methods for information retrieval from highly sparse data such as capsule network and attention mechanism. The mathematical model is abstracted by Guo et al. [4] on the basis of the production scheduling problem. According to the different parts of the same machine and the different processes of the same part, the corresponding processing time and waiting time are obtained. Song et al. [5] described the multimodal model of machine learning research status and research significance of the text on the exoskeleton robot applications and on the basis of a detailed study of gait. With the rapid expansion of data scale, the traditional method of air quality prediction technology has been unable to deal with these massive data. Three hundred and sixty-five sets of air pollutant data from January 1, 2018, to December 31, 2018, in Shen Zhen were used by Sun et al. [6] as experimental objects. Lian et al. [7] proposed an accelerated matrix decomposition mechanism, which could be used to boost not only the original Hessian-based multi-scale approach but also the singular value decomposition-based algorithms. Zhu and Zheng [8] studied the application of support vector machine in traffic identification to classify the network traffic. Shi et al. [9] develop a new rainy image model to describe rain scenes at night with low illumination.

Support vector machine (SVM) model method is used by Fan et al. [10] to classify and predict different disease processes of Alzheimer's disease based on structural brain magnetic resonance imaging (MRI) data, so as to help the 
auxiliary diagnosis of the disease. Accurate recognition of the type of ground motion is a basic task in the field of seismic engineering. The key technologies of detection and recognition of underground seismic signal are studied by Zhong and $\mathrm{Li}$ [11]. An unsupervised co-segmentation algorithm is proposed by Zhang et al. [12], which can be applied to the image with multiple foreground objects simultaneously and the background changes dramatically. In order to realize the automation of radar signal recognition by machine learning, Li [13] proposes an automatic machine learning AUTO-SKLEARN system and applies it to radar radiation source signals. In order to make up for the gap in the auxiliary diagnosis of orthopedics and promote the wisdom process of orthopedic disease diagnosis, $\mathrm{Li}$ and Zhang [14] proposed an orthopedic auxiliary classification prediction model based on XGBoost algorithm. In order to improve the efficiency of hospital drug inventory management, based on genetic algorithm and BP neural network, Luo et al. [15] combined the actual situation of hospital drug inventory forecast to build a system model based on hospital drug management mode. Different from the existing algorithms which are often based on heuristic algorithms, Wang et al. [16] proposed a VNE algorithm for data center topology based on the Q-learning algorithm which is a typical reinforcement learning method. Li et al. [17] analyze the low-carbon integrated forward/reverse logistics network and made relevant simulation tests. $\mathrm{Xu}$ and Jiang [18] proposed a short-term traffic flow forecasting method based on deep belief network-support vector regression. Chen et al. [19] undertake such analysis using a deep convolutional neural network algorithm named AlexNet.

Acknowledgements The guest editors would like to thank Prof. John MacIntyre who is the editor in chief of Neural Computing and Applications. His help and trust are the most important things for the success of this SI. The guest editors would like to thank the reviewers for their high-quality reviews, which provided insightful and constructive feedback to the authors of the papers.

\section{References}

1. Wei P, He F, Zou Y (2020) Content semantic image analysis and storage method based on intelligent computing of machine learning annotation. Neural Comput Appl. https://doi.org/10. 1007/s00521-020-04739-4

2. Zhong X, Liu J, Li L et al (2019) An emotion classification algorithm based on SPT-CapsNet. Neural Comput Appl. https:// doi.org/10.1007/s00521-019-04621-y

3. Jain DK, Jain R, Upadhyay Y et al (2019) Deep refinement: capsule network with attention mechanism-based system for text classification. Neural Comput Appl. https://doi.org/10.1007/ s00521-019-04620-Z

4. Guo K, Yang M, Zhu H (2019) Application research of improved genetic algorithm based on machine learning in production scheduling. Neural Comput Appl. https://doi.org/10.1007/ s00521-019-04571-5

5. Zheng Y, Song Q, Liu J et al (2019) Research on motion pattern recognition of exoskeleton robot based on multimodal machine learning model. Neural Comput Appl. https://doi.org/10.1007/ s00521-019-04567-1

6. Gu K, Zhou Y, Sun H et al (2019) Prediction of air quality in Shenzhen based on neural network algorithm. Neural Comput Appl. https://doi.org/10.1007/s00521-019-04492-3

7. Zhang Y, Lian J, Rong L et al (2019) Even faster retinal vessel segmentation via accelerated singular value decomposition. Neural Comput \& Applic. https://doi.org/10.1007/s00521-01904505-1

8. Zhu Y, Zheng Y (2019) Traffic identification and traffic analysis based on support vector machine. Neural Comput Appl. https:// doi.org/10.1007/s00521-019-04493-2

9. Shi Z, Feng Y, Zhao M et al (2019) A joint deep neural networksbased method for single nighttime rainy image enhancement. Neural Comput Appl. https://doi.org/10.1007/s00521-019-045015

10. Fan Z, Xu F, Qi X et al (2019) Classification of Alzheimer's disease based on brain MRI and machine learning. Neural Comput Appl. https://doi.org/10.1007/s00521-019-04495-0

11. Zhong Z, Li H (2019) Recognition and prediction of ground vibration signal based on machine learning algorithm. Neural Comput Appl. https://doi.org/10.1007/s00521-019-04496-Z

12. Zhang L, Sheng Z, Li Y et al (2019) Image object detection and semantic segmentation based on convolutional neural network. Neural Comput Appl. https://doi.org/10.1007/s00521-019-044914

13. Li P (2019) Research on radar signal recognition based on automatic machine learning. Neural Comput Appl. https://doi. org/10.1007/s00521-019-04494-1

14. Li S, Zhang X (2019) Research on orthopedic auxiliary classification and prediction model based on XGBoost algorithm. Neural Comput Appl. https://doi.org/10.1007/s00521-019-04378-4

15. Du M, Luo J, Wang S et al (2019) Genetic algorithm combined with BP neural network in hospital drug inventory management system. Neural Comput \& Appl. https://doi.org/10.1007/s00521019-04379-3

16. Yuan Y, Tian Z, Wang C et al (2019) A Q-learning-based approach for virtual network embedding in data center. Neural Comput Appl. https://doi.org/10.1007/s00521-019-04376-6

17. Ren Y, Wang C, Li B et al (2019) A genetic algorithm for fuzzy random and low-carbon integrated forward/reverse logistics network design. Neural Comput Appl. https://doi.org/10.1007/ s00521-019-04340-4

18. Xu H, Jiang C (2019) Deep belief network-based support vector regression method for traffic flow forecasting. Neural Comput Appl. https://doi.org/10.1007/s00521-019-04339-x

19. Li S, Chen J, Xiang J (2019) Applications of deep convolutional neural networks in prospecting prediction based on two-dimensional geological big data. Neural Comput Appl. https://doi.org/ 10.1007/s00521-019-04341-3

Publisher's Note Springer Nature remains neutral with regard to jurisdictional claims in published maps and institutional affiliations. 